\title{
Topological States in a Segmented Chain
}

\section{A segmented chain of molecules held together by van der Waals forces may host spin-polarized, topologically protected electron states.}

\section{by Jerzy Bernholc ${ }^{1}$}

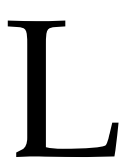

ow-dimensional materials are at the forefront of condensed-matter physics research. In these materials, the quantum confinement of electrons leads to unique properties, including the emergence of topological electronic states. Electrons in these states are protected from scattering off other electrons, which could lead to materials in which electronic conduction is not affected by atomic imperfections. Two materials that have attracted much recent attention because of their topological properties are single layers of graphite (graphene) and transition-metal chalcogenides (TMCs) - atomically thin semiconductors that comprise a transition metal and a sulfur-like element. However, it is unclear what conditions cause a material to exhibit topological protection as its dimensions are reduced. Through experiments and calculations, a team led by Alex Zettl and Marvin Cohen of the University of California, Berkeley, and Lawrence Berkeley National Laboratory has engineered a novel one-dimensional TMC structure: a linear chain made of several segments bonded end-to-end by weak van der Waals (vdW) forces. The researchers' calculations show that the material may be further tweaked to exhibit topological properties [1].

Many low-dimensional materials form spontaneously, as is the case for graphene or the above-mentioned layered structures of TMC semiconductors (which have chemical formula $M X_{2}$, where $M$ is a transition metal and $X$ is a chalcogen: sulfur, selenium or tellurium). However, fabricating a chain made of multiple segments is more difficult than forming an atomically thin sheet. Researchers have found that by placing atoms inside a hollow carbon nanotube, they can synthesize novel 1D structures that are protected from uncontrolled interactions with atoms in their environment. Recent demonstrations with this nanotube approach include structures that can be viewed as 1D phases of individual elements [2] or compounds [1, 3, 4]. The Berkeley group synthesized 1D TMCs by confin-

${ }^{1}$ Department of Physics, North Carolina State University, Raleigh, NC, USA
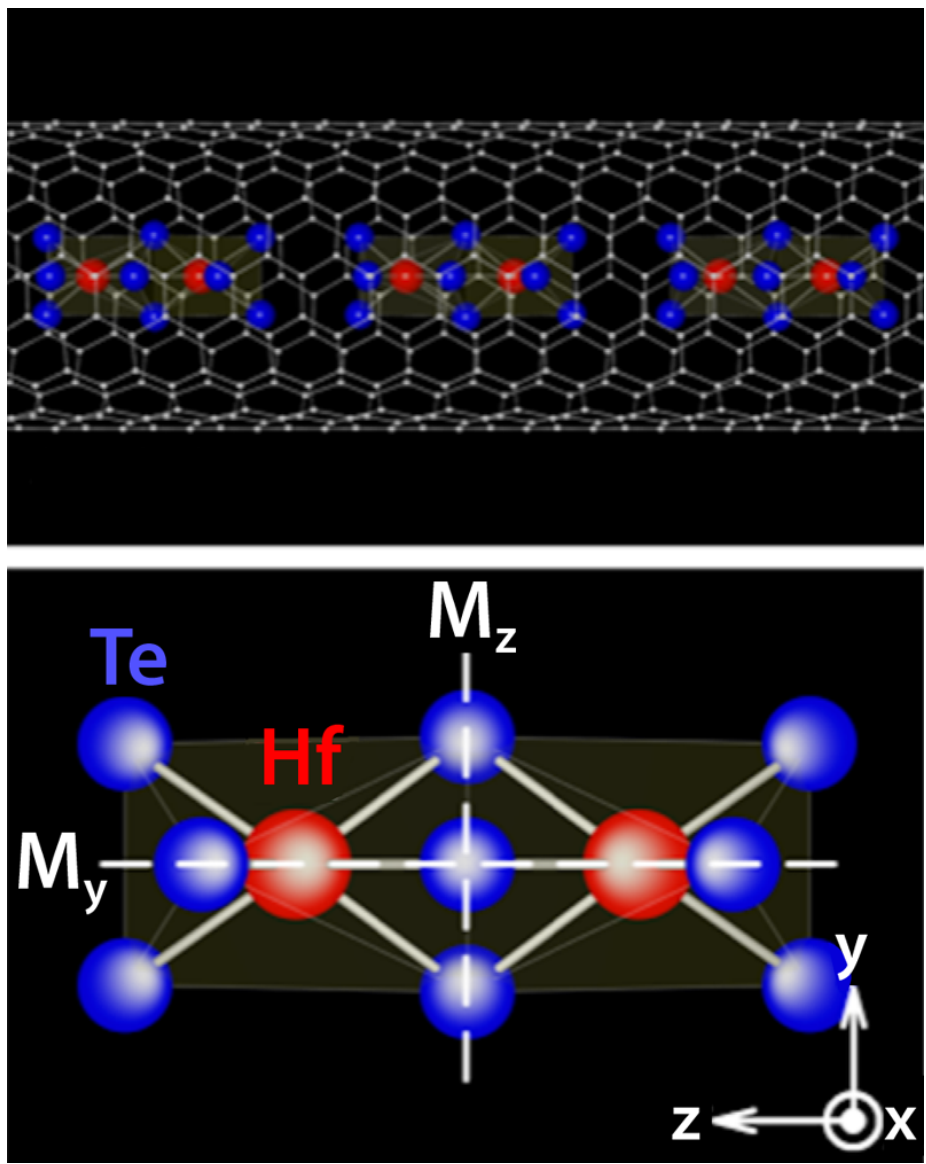

Figure 1: (Top) Sketch of the nanotube-encapsulated segmented chain demonstrated by Zettl, Cohen, and co-workers [1]. The molecules in the chain form well-separated segments that are bonded by van der Waals forces. (Bottom) Structure of the $\mathrm{Hf}_{2} \mathrm{Te}_{3}$ molecules, which have two mirror-symmetry planes, $M_{y}$ and $M_{z}$, perpendicular to the $y$ and $z$ axes, respectively. (T. Pham et al. [1])

ing the components-an appropriate mixture of hafnium (Hf) and tellurium (Te) atoms-inside the nanotubes. As a result, never-before-seen periodic structures formed within the nanotubes.

To understand the structure of their material and its emer- 


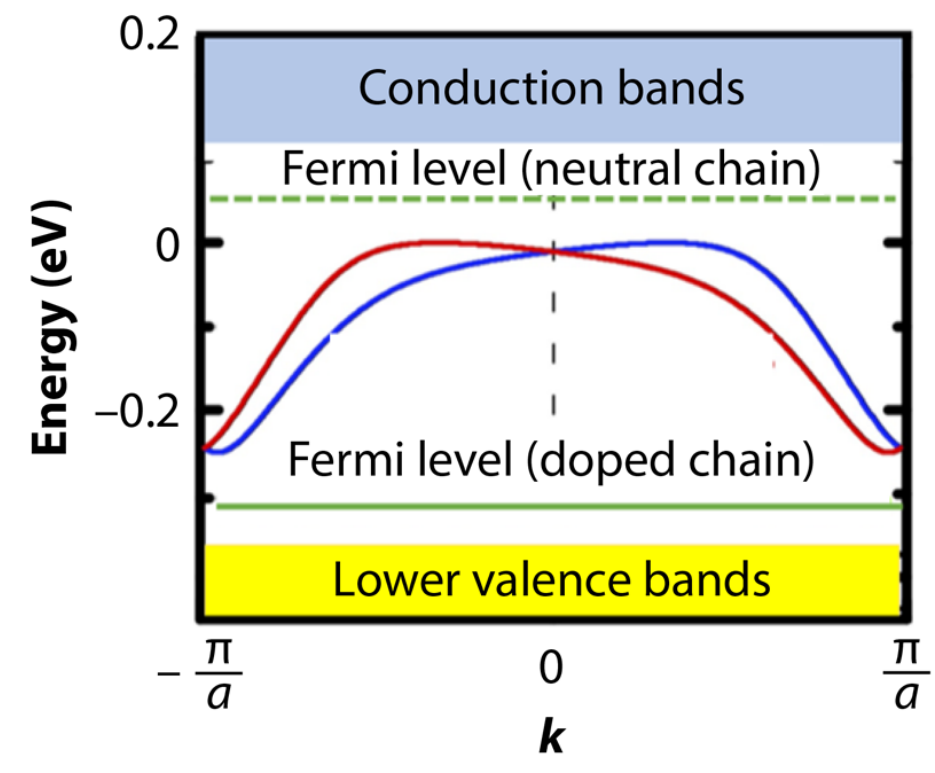

Figure 2: Segmented-chain energy-band structure near the Fermi level. In the top two valence bands (plotted in red and blue), electrons move in opposite directions, resulting in a topologically nontrivial time-reversal pair. When the chain is hole doped, the Fermi level shifts, and the material becomes a "topological mirror" insulator. (T. Pham et al. [1]; adapted by Jerzy Bernholc)

gent electronic properties, the team combined atomically resolved scanning transmission electron microscopy (STEM) with theoretical and numerical analysis. Using state-of-theart density-functional theory, they identified several possible candidate structures and predicted that the most likely arrangement was one in which each segment had the same $\mathrm{Hf}_{2} \mathrm{Te}_{9}$ structure. They confirmed this conclusion by showing, with simulations, that the calculated STEM images for segments with this structure provided the best fit to the experimental images. $\mathrm{The}_{2} \mathrm{Hf}_{2} \mathrm{Te}_{9}$ segments are well separated from each other and thus can only bond via van der Waals forces (Fig. 1).

Further analysis indicated that a completely unexpected topological state may emerge from the weakly bound molecular chain. The presence of hafnium plays an important role in this topological behavior. Being a heavy metal, hafnium has an intense electric field near its nucleus, which accelerates electrons to relativistic speeds and couples their orbital angular momentum to their spin. This spin-orbit coupling, which acts like a magnetic field that deflects electrons with opposite spins in opposite directions, is a key ingredient in the emergence of topologically protected states.

The theoretical analysis provides the following picture for the bands available to electrons moving within each segment. Each $\mathrm{Hf}_{2} \mathrm{Te}_{9}$ segment is highly symmetric, with two mirror planes. The combined symmetries lead to the chain's unique electronic band structure. Along the $z$ direction, the periodicity of the chain results in a sequence of 1D energy bands that relate electron momenta and energies. When strong spin-orbit coupling is present, the energies of spinup and spin-down electrons, which are usually the same, split into pairs [5]. The energy bands are mirror symmetric around zero crystal momentum, but the electrons in one band move in a direction opposite to those in the other, as required by time-reversal symmetry (Fig. 2).

The mirror symmetry in the $M_{z}$ plane leads to a welldefined topological phase in each 1D energy band, called a Zak phase [6]. The Zak phases determine, through a quantity called the symmetry-protected topological invariance, whether a given band is topologically trivial (normal) or topologically nontrivial [5]. Topological invariance is calculated as the sum of the Zak phases of the occupied bands in which electrons move in one direction, quantifying how the electron wave functions of the bands interfere with each other. If the sum is not 0 , the band structure results in a topological insulator. Mathematically, one can show that if the charge at the end of a finite-length polarized chain contains an odd number of electrons, the chain is a topological insulator $[7,8]$.

The researchers' calculations show that the topological invariance for the chain is 0 , meaning that the material is not a topological insulator. While the top two valence bands are topologically nontrivial, they are separated from the other bands by large gaps. The researchers propose that the topological nature of these bands may be realized by adding positive carriers, or holes, by, for example, providing electron-deficient atoms nearby. The conducting electrons would then be moving in the topological states. The researchers performed explicit calculations for a hole-doped, 15-nm-long chain, predicting the odd charge at the end of the chain that indicates the topological insulator behavior.

This proposal offers an unexpected way to create a new topological material with protected electron conduction. The engineering of this material may provide a new paradigm for the design and synthesis of other 1D materials with symmetry-protected states. Future designs may not need to encapsulate the atoms in carbon nanotubes. Rather, the molecules may be coerced to directly assemble, through sophisticated chemical or physical procedures, into configurations that could serve as elements of spintronic nanodevices, topological transistors, or qubits.

This research is published in Physical Review Letters.

\section{REFERENCES}

[1] T. Pham et al., "Emergence of topologically nontrivial spinpolarized states in a segmented linear chain," Phys. Rev. Lett. 124, 206403 (2020).

[2] T. Fujimori et al., "Formation and properties of selenium doublehelices inside double-wall carbon nanotubes: Experiment and theory," ACS Nano 7, 5607 (2013). 
[3] T. Pham et al., "Torsional instability in the single-chain limit of a transition metal trichalcogenide," Science 361, 263 (2018).

[4] S. Meyer et al., "Metal-insulator transition in quasi-onedimensional $\mathrm{HfTe}_{3}$ in the few-chain limit," Phys. Rev. B 100, 041403 (2019).

[5] L. Fu and C. L. Kane, "Time reversal polarization and a $Z_{2}$ adiabatic spin pump," Phys. Rev. B 74, 195312 (2006).

[6] J. Zak, "Berry's phase for energy bands in solids," Phys. Rev.
Lett. 62, 2747 (1989).

[7] A. Lau et al., "Topological mirror insulators in one dimension," Phys. Rev. B 94, 165164 (2016).

[8] G. van Miert and C. Ortix, "Excess charges as a probe of onedimensional topological crystalline insulating phases," Phys. Rev. B 96, 235130 (2017).

10.1103/Physics. 13.82 